\title{
Relation between Single Nucleotide Polymorphism rs3738423 (C $>$ T) of NPHS2 Gene and some Biochemical Parameters in Pediatrics Nephrotic Syndrome Patients
}

\author{
Nga Van VU ${ }^{1}$, Long Doan DINH ${ }^{1}$, Nhung Thi Hong PHAM ${ }^{1}$, \\ Dem Van PHAM', Quy Van DAM ${ }^{1}$, Huong Thi Quynh NGUYEN ${ }^{2}$, \\ Thinh Huy TRAN ${ }^{2}$ and Thom Thi VU, ${ }^{1, *}$ \\ ${ }^{1}$ School of Medicine and Pharmacy, Vietnam National University, Cau Giay, Ha Noi, Viet Nam \\ ${ }^{2}$ Hanoi Medical University, Kim Lien, Dong Da, Ha Noi, Viet Nam
}

('Corresponding author's e-mail: thomtbk5@gmail.com)

Received: 6 March 2019, Revised: 28 November 2019, Accepted: 12 December 2019

\begin{abstract}
Clinical biochemical parameters were known as important criteria for diagnosis and treatment of nephrotic syndrome (NS). Single nucleotide polymorphism (SNP) rs3738423 (C>T) of NPHS2 gene encoding for podocin protein in globular membrane was proved associated to steroid resistant nephrotic syndrome. This study was to evaluate the relation between rs3738423 (C>T) of NPHS2 gene and biochemical parameters of pediatrics NS patients in steroid- sensitive (SS), early (ESR) and late (LSR) steroid resistant groups. Blood and urine samples from 149 patients were collected for protein, albumin and protein/creatinine analysis; blood samples in EDTA tube were used for NPHS2 gene analysis. The results showed that CC and CT genotypes were mostly abundant in all three groups, whereas TT was minor appeared in only SS and KTM groups. Patients with the CT genotypes in the ESR group had a protein/creatinine index nearly twice as high as those patients with the CC genotypes. Meanwhile, those with CT genotypes in the SS and LSR groups had lower rates than the CC genotypes. Clinical biochemical parameters significantly contributed to the diagnosis, monitoring and treatment of pediatrics nephrotic syndrome, the changes of these parameters depended on the interaction between the response to corticoid and rs3738423 (C>T) polymorphism of NPHS2 gene in pediatrics nephrotic syndrome patients.
\end{abstract}

Keywords: Biochemical parameters, NPHS2, rs3738423 (C>T), Pediatrics nephrotic syndrome

\section{Introduction}

Childhood nephrotic syndrome is a clinical and biochemical syndrome related to glomerular injury caused by a variety of pathologies. It is the most common nephropathy in children. Incidence rates vary by geography, age, sex and race. At Vietnam National Children's Hospital, the amount of nephrotic syndrome patients is about $0.5-1 \%$ of the total number of inpatients and about $10-30 \%$ of all kidney disease cases [12]. Approximately $20 \%$ of patients do not respond to corticosteroids and other immunosuppressive drugs, $50 \%$ of these patients will progress to end-stage renal failure, which impact much on health and life [7].

In addition to clinical signs such as rapid body edema, hematuria, some biochemical tests are very important to diagnosis and monitoring including proteinuria, blood proteins, blood albumin, blood urea, blood creatinine, lipid and cholesterol. Nephrotic syndrome is a chronic disease, with the spontaneous outbreaks, patients should be monitored for a long time (at least 5 years after remission) [12].

Advances in molecular genetics have identified genes that code for proteins that play an important role in glomerular function, including podocin [14]. Podocin is a membrane protein which is encoded by NPHS2 gene located in chromosome 1 (1q25 - q31) with 8 exons. The research show that the NPHS2 mutation, in 
http://wjst.wu.ac.th

particular the $288 \mathrm{C}>\mathrm{T}$ ( $\mathrm{S} 96 \mathrm{~S}$ or $\mathrm{rs} 3738423$ ) polymorphism was a substitution of $\mathrm{C}=>\mathrm{T}$ at nucleotide 16170in exon 2, is closely related to primary corticosteroid resistance [1]. Study of Junli showed 7 heterozygous variants and 1 homozygous variant of SNP $288 \mathrm{C}>\mathrm{T}$. This study also found that SNP $288 \mathrm{C}>\mathrm{T}$ may have a protective effect in Chinese patients but there was no association observed for the Malaysian patients [4]. In Vietnam, there is no report on the correlation between NPHS2 single nucleotide polymorphism and some biochemical results in childhood nephrotic syndrome. Therefore, this study could initially help clinicians better in diagnosis and monitor patients, especially in steroid-resistant group so patients do not have to suffer from severe complications and side effects of immunosuppressive drugs. We decided to evaluate the relationship between $\mathrm{rs} 3738423(\mathrm{C}>\mathrm{T})$ of gene NPHS2 and some biochemical parameters in children with nephrotic syndrome in 3 groups: steroid-sensitive nephrotic syndrome (SSNS), early steroid-resistant nephrotic syndrome (ESRNS), late steroid-resistant nephrotic syndrome (LSRNS).

\section{Materials and methods}

\section{Patients and control}

We enrolled 149 patients in the Departement of Kidney and Urology from the Vietnam National Children's Hospital from 2015 to 2016, was diagnosed with KDIGO (Kidney Disease Improving Global Outcomes) in 2012 including proteinuria $\geq 50 \mathrm{mg} / \mathrm{kg} / 24 \mathrm{~h}$ or proteinuria/cretinineuria index $\geq 200$ $\mathrm{mg} / \mathrm{mmol}$, blood albumin $\leq 25 \mathrm{~g} / \mathrm{L}$, blood protein $\leq 56 \mathrm{~g} / \mathrm{L}$. Based on the response to the initial standard steroid treatment (4 weeks with prednisolone $2 \mathrm{mg} / \mathrm{kg} /$ day, complete remission blood protein $>56 \mathrm{~g} / \mathrm{L}$, blood albumin $>25 \mathrm{~g} / \mathrm{L}$ ) and steroid resistance (no improvement after 6 weeks of prednisolone $2 \mathrm{mg} / \mathrm{kg} / \mathrm{day}$ daily with clinical symptom such as: proteinuria $\geq 50 \mathrm{mg} / \mathrm{kg} / 24 \mathrm{~h}$ or proteinuria/creatinineuria index $\geq 200$ $\mathrm{mg} / \mathrm{mmol}$, blood albumin $\leq 25 \mathrm{~g} / \mathrm{L}$, blood protein $\leq 56 \mathrm{~g} / \mathrm{L}$ ) or no improvement after 4 weeks with prednisolon $2 \mathrm{mg} / \mathrm{kg} /$ day for 4 consecutive days and $2 \mathrm{mg} / \mathrm{kg} /$ day for prednisolone daily or 3 doses of methylprednisolone $1000 \mathrm{mg} / 1,73 \mathrm{~m}^{2} / 48 \mathrm{~h}$. Patients who is corticosteroid resistance at the first treatment is early steroid-resistant nephrotic syndrome, the case of resistance after the first treatment is called late steroid-resistant nephrotic syndrome.

The study was approved by scientific committee of Vietnam National University Hanoi as well as ethics committee of Vietnam National Pediatrics Hospital coding number 796/BVNTW-VNCSKTE and agreement consent of all patients and their parents were collected.

\section{Sampling and preservation methods}

$24 \mathrm{~h}$ urine collection biochemistry: take all urine in one day and night $(24 \mathrm{~h})$. Urine container must be covered, washed and sterilized with $5 \mathrm{ml}$ of concentrated $\mathrm{HCl}$. In the evening before, shower, clean the urinary genitalia. At 6 am, patients don't save the urine from the first time urinating, began to note the time on the container as the start time. All urine, after the first flushed specimen until 6 am in the next morning, must be saved, stored and kept cold in the container. Measure urine $24 \mathrm{~h}$, take $5 \mathrm{ml}$ for testing. Preserve urine with $10 \%$ thymol solution $(5 \mathrm{ml})$. Biochemical blood sample: Take morning blood, not eat. Take $1.5-2 \mathrm{ml}$ intravenous blood in the anti-coagulation with Heparin. The specimens were transported to the laboratory for analysis. Sample for genetic analysis: Collect $2 \mathrm{ml}$ of whole blood in an EDTA anticoagulation tube, stored at $-20{ }^{\circ} \mathrm{C}$ until use.

\section{Quantitative biochemistry analysis of blood and urine}

The samples were analyzed at the Department of Biochemistry, Vietnam National Pediatrics Hospital. Patients were tested at different time points: hospitalization, discharge, 1 month after discharge and 6 months after discharge. The basic biochemical parameters analyzed including proteinuria, proteinuria/creatininuria index, blood proteins, blood albumin, blood creatinine using AU2700 (Beckman Coulter). 
http://wjst.wu.ac.th

\section{NPHS2 gene polymorphisms analysis}

DNA was extracted from whole blood samples in EDTA anti-coagulation using E.Z.N.A blood DNA Mini kit (Omega-Biotek). The primer of exon 2 was designed and evaluated using the PerlPrimer vesion 1.1.14 software was synthesized at ISDT (America). The order of all primers was published [6]. Gene sequences carrying exons are cloned by polymerase chain reaction (PCR). A $1.5 \%$ agarose gel is used for the analysis of PCR products, using the Ruler 100bp Plus DNA Ladder (SM0321, Thermo Scientific) and images taken using Gel-Doc It (Applied Biosystem) to test the quantity of products. PCR products were then purified using the E.Z.N.A.Acycle-Pure Kit (Omega-Biotek) and the 3500 Applied Biosystems and the BigDye ${ }^{\circledR}$ Terminator v3.1 cycle sequencing kit (Applied Biosystems). The sequencing results were analyzed using BioEdit version 7.1.9 software, thereby identifying the patient's genotype.

\section{Statistical analysis}

Data were analyzed by SPSS 16.0 software. Analysis of variance (ANOVA) of 1 factor and 2 factors; Chi-square tests $(\chi 2)$ are applied to the corresponding cases. p-value of less than 0.05 was viewed as a statistically significant difference.

\section{Results and discussion}

In nephrotic syndrome, in addition to clinical symtomps such as hypertension, edema, subclinical and urinary indices such as proteinuria, proteinuria/creatinineuria index, blood proteins, blood albumin plays an important role in diagnosis, monitoring and treatment. Patients were divided into 3 groups based on corticosteroid response: steroid-sensitive nephrotic syndrome (SSNS), early steroid-resistant nephrotic syndrome (ESRNS), late steroid-resistant nephrotic syndrome (LSRNS), vertical tracking at multiple points, hospital, discharge, one month after discharge and 6 months after discharge. Urinalysis results are shown in Figure 1.

A

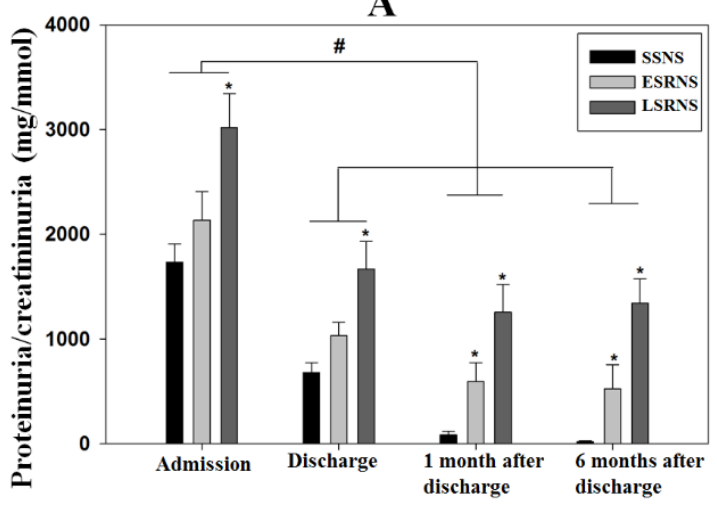

B

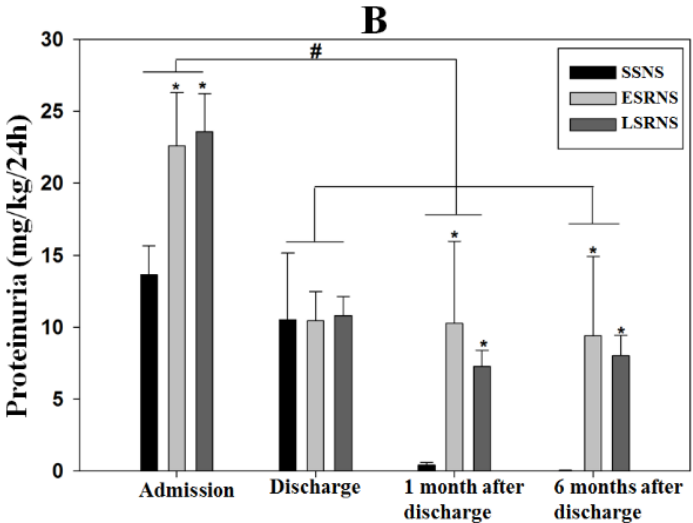

Figure 1 Proteinuria/creatininuria index (Figure 1A) and mean proteinuria (Figure 1B) in the SSNS, ESRNS and LSRNS groups at time of admission, discharge, 1 month and 6 months after discharge. The sign * shows a significant difference between the two groups of ESRNS and LSRNS compared with the SSNS group: *p $<0.05$; \# between the time of hospitalization and 3 points: discharge, after 1 month, after 6 months difference: $\mathrm{p}<0.05$.

In Figure 1, the proteinuria/creatininuria index (Figure 1A) and proteinuria (Figure 1B) tended to decrease over time, from the time the patient was admitted to hospital to 6 months after discharge. Figure 1A shows that the proteinuria/creatininuria index in SSNS group is lower than that of the ESRNS and LSRNS with $\mathrm{p}$ value $<0.05$. In addition, the rate in the LSRNS group was lower than that in 3 points: hospitalization, discharge and one month after discharge with p values: $0.012 ; 0.207 ; 0.042$, respectively. Figure B shows that 
http://wjst.wu.ac.th

at the time of admission, the proteinuria of the SSNS $(14.02 \pm 1.10 \mathrm{mg} / \mathrm{kg} / 24 \mathrm{~h})$ was lower than the ESRNS group $(23.39 \pm 2.31 \mathrm{mg} / \mathrm{kg} / 24 \mathrm{~h})(\mathrm{p}=0.003)$. After 1 month and 6 months of discharge from hospital, the patients with proteinuria were lower than LSRNS group with $p=0.033$ and $p=0.028$, respectively.

In nephrotic syndrome, urinary biochemistry and blood biochemistry are indispensable indicators for monitoring and treatment of diseases. Therefore, biochemical indicators such as protein, albumin are also recorded. This difference in blood levels among the patient groups at different times is shown in Figure 2.

A

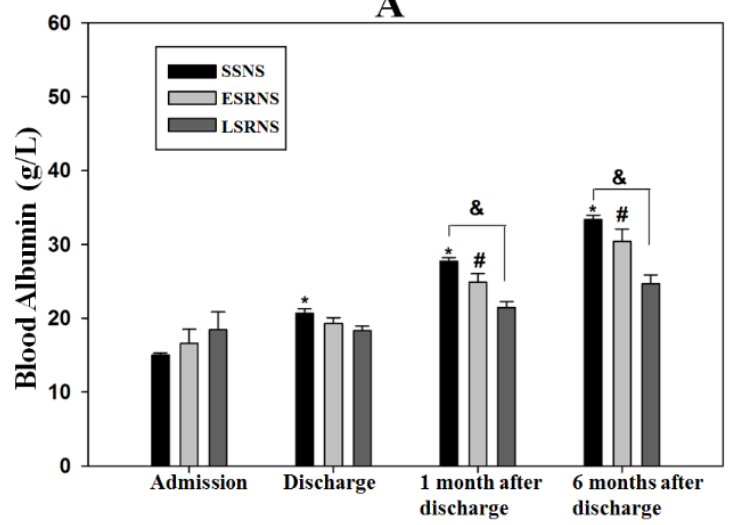

B

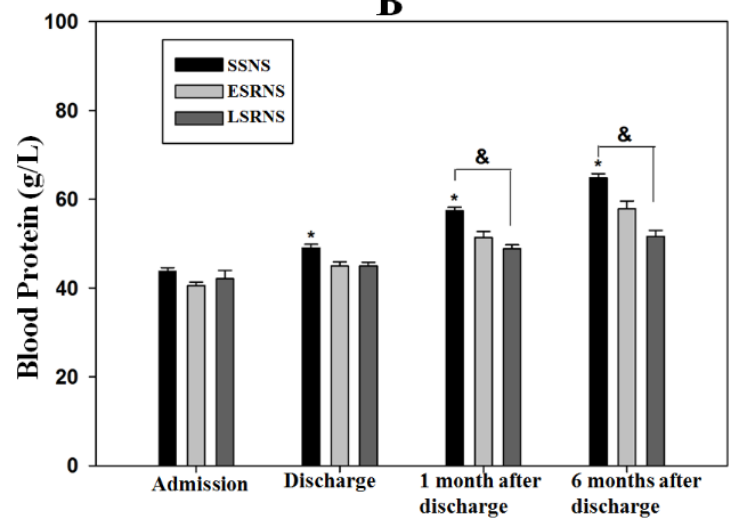

Figure 2 Blood albumin (Figure 2A) and blood protein concentration (Figure 2B) in the SSNS, ESRNS and LSRNS groups at time of admission, discharge, 1 month and 6 months after discharge. The sign * shows a significant difference between the time of admission of SSNS group and discharge time, one month and 6 months after discharge: $\mathrm{p}<0.05$; \# showed that the LSRNS group at 1 month and 6 months significantly different from the time of hospitalization: $\mathrm{p}<0.05$ and shows the difference between the SSNS and ESRNS at 1 month and 6 months after discharge.

Both blood albumin (Figure 2A) and blood protein (Figure 2B) concentration tended to increase over time. At the time of admission, both these two indexes among the three groups were not different. However, at 3 points time: discharge, 1 month and 6 months after discharge. SSNS group had higher blood protein concentration than ESRNS group with $\mathrm{p}$ value $=0.000$. In addition, blood protein concentration in the LSRNS group was higher than those in the 1 month and 6 months after discharge of ESRNS group with $\mathrm{p}$ value 0.012 and 0.004 , respectively. The blood protein concentration in LSRNS group was also significantly lower than that of the SSNS group at discharge point, 1 month and 6 months after discharge with $\mathrm{p}$ value $0.027 ; 0.000 ; 0.004$, respectively. Similarly, blood albumin concentration was significantly lower in ESRNS group than in SSNS and LSRNS group with $p$ value $=0.000$.

Prior to treatment, proteinuria, proteinuria/creatininuria index in both SSNS and corticosteroid resistance groups were very high. After treatment, proteinuria and proteinuria/creatininuria index in all groups were lower than before treatment. However, at all times before or after treatment, the concentration of these substances in the urine of the corticosteroid resistance group was always higher than the sensitivity group. Similar results were observed in the protein and blood albumin concentrations but in the opposite trend. Blood protein and albumin concentrations before treatment were low and there was no statistically significant difference between the groups. Inversely proportional with substances in the urine, concentration of these substances in the blood gradually increase with the duration of treatment and the blood protein and albumin concentrations in the corticosteroid resistance group is lower than the sensitive group with statistically significant. This result is consistent with the results in previous studies $[7,10,11]$. It is notable that between the two patient's groups with early resistance and late resistance, blood albumin and protein concentrations were different at all post-treatment points. This phenomenon 
http://wjst.wu.ac.th

has not been observed in Indian patients but appears in our study in Vietnamese patients and in other studies in Bangladesh [5,10].

The presence of biochemical index such as protein and albumin in urine and the decline of these indexes in the blood reflect the decreased renal filtration and renal function. Podocin is a protein located on glomerular filtration membrane, coded by the NPHS2 gene, which is thought to be related to clinical and paraclinical symptom in nephrotic syndrome. Thus, in addition to monitoring biochemical indexes, patients were also identified the SNP rs3738423 $(\mathrm{C}>\mathrm{T})$ at exon 2 of the NPHS2 gene. The results are presented in Table 1.

Table 1 Genotype ratio, allele frequencies of rs3738423(C>T) of exon 2 of NPHS2 in 3 groups: steroidsensitive nephrotic syndrome (SSNS), early steroid-resistant nephrotic syndrome (ESRNS), late steroidresistant nephrotic syndrome (LSRNS).

\begin{tabular}{ccccc}
\hline \multirow{2}{*}{ rs3738423 $(\mathbf{C}>\mathbf{T})$ of exon 2 } & \multicolumn{2}{c}{ Three groups of corticosteroid response } \\
\cline { 2 - 4 } & $\mathbf{C C}$ & SSNS & ESRNS & LSRNS \\
\cline { 2 - 4 } Genotype & $\mathbf{C T}$ & $17(81.03 \%)$ & $43(76.79 \%)$ & $28(80.00 \%)$ \\
& TT & $1(1.73 \%)$ & $13(23.21 \%)$ & $6(17.14 \%)$ \\
& $\mathbf{p}$ & 0.9 & - & $1(2.86 \%)$ \\
\hline \multirow{2}{*}{ Allen } & $\mathbf{C}$ & 0.1 & $\chi^{2}=2.13 ; \mathrm{p}=0.75$ & \\
frequencies & $\mathbf{T}$ & & $\chi^{2}=0.20 ; \mathrm{p}=0.90$ & 0.11 \\
& $\mathbf{p}$ & & & \\
\hline
\end{tabular}

In 149 patients shown in Table 1, rs3738423 (C>T) of exon 2 predominate in the CC and CT genotypes. The TT genotype was very small, in the SSNS and LSRNS groups. Genotype ratios in all three groups were not significantly different $p$ value $=0.75$. Allele frequencies of $r s 3738423(C>T)$ are distributed according to Hardy-Weinberg's law with the rate of alleles $C$ in the 3 group SSNS: ESRNS: LSRNS: 0.90: 0.88: 0.89, respectively. There was no difference in frequency of alleles observed in these three groups with $\mathrm{p}=0.90$.

Changes in blood and urine biochemical parameters in nephrotic syndrome patients are not only affected by corticosteroid responses but are also influenced by interactions between these factors and the genotypes shown in Table 2.

Table 2 Proteinuria/creatininuria index according to the corticosteroid response and genotype of rs3738423 C $>\mathrm{T}$ in exon 2 of NPHS2.

\begin{tabular}{lccccc}
\hline \multirow{2}{*}{$\begin{array}{c}\text { Biochemical index } \\
(\bar{X} \pm \text { SE) }\end{array}$} & \multirow{2}{*}{ Group } & \multicolumn{2}{c}{ Genotype of rs3738423 (C>T) on exon 2 } & p value \\
\cline { 3 - 5 } & & CC & TC & TT & 0.011 \\
\hline \multirow{2}{*}{$\begin{array}{l}\text { Proteinuria/creati-ninuria } \\
\text { index in time of }\end{array}$} & SSNS & $1827.70 \pm 132.64$ & $1441.90 \pm 128.39$ & $1984.00 \pm 0.00$ \\
addmision (mg/mmol) & ESRNS & $2829.97 \pm 285.67$ & $4336.75 \pm 835.04$ & - & $1142.00 \pm 0.00$ \\
\hline Proteinuria/creati-ninuria & SSRNS & $2295.50 \pm 289.00$ & $1223.83 \pm 340.85$ & $1423.00 \pm 0.00$ \\
index in discharge time & ESRNS & $1334.72 \pm 131.41$ & $2872.42 \pm 874.77$ & - & 0.001 \\
$(\mathbf{m g} / \mathbf{m m o l})$ & LSRNS & $1119.92 \pm 127.55$ & $653.83 \pm 185.05$ & $10.30 \pm 0.00$ & \\
\hline
\end{tabular}


http://wjst.wu.ac.th

Table 2 shows the interaction between corticosteroid response and genotype SNP rs3738423 C>T in exon 2 of NPHS2, which changes the proteinuria/creatininuria index at admission and discharge time with $\mathrm{p}$ values of 0.011 and 0.001 , respectively. Individuals carry a mutant allele (Heterozygous) in ESRNS groups who had a high proteinuria/creatininuria index were nearly twice as likely to these group with type of homozygous. In contrast, in the SSNS group and the LSRNS group, the index was lower in those with heterozygous versus wild type combinations.

Many studies have shown that polymorphism, even when not substituted for amino acids, can lead to phenotypic changes by affecting the structure of the mRNA or by inactivation of the gene through connected genes to bypass the mutant exon [6]. Therefore, polymorphism may play a role in the development of the primary childhood nephrotic syndrome and may also lead to different phenotypes in patients. In our study, rs3738423 (C>T) in the two groups were consistent with Hardy-Weinberg's law. Data shows that stability has been achieved in the population. The frequency of allele $\mathrm{C}$ in the SSNS group was 0.9; in the LSRNS group is 0.89 and in the ESRNS there is no difference. When compared to the frequency of allele $\mathrm{C}$ in other patient populations around the world, the results show that the frequency of alleles in our study was smaller than in other studies. Our study is different from Jun Li's 2012 study of 97 patients with primary nephrotic syndrome with a frequency of 0.95 [4]. Caridi's study on 44 patients who had been diagnosed with FSGS also had a higher C allele of 0.94 [2]. Although no differences in genotyping and allele frequency among groups in many studies [3,9,13]. Jun Li's study (2012) showed that rs3738423 (C>T) seems to protect Chinese patients. Allele frequencies were lower in the patient group $(5 \%)$ than in the control group $(13 \%)$. Protective effects have not been observed in other studies in patients from northern and eastern China. The discrepancies between studies on Chinese patients may be due to either the allele frequencies or genetic variations between North and South China. The results of our study in Table 2 show that $\mathrm{rs} 3738423(\mathrm{C}>\mathrm{T})$ have a protective effect on the reduction of proteinuria/creatinine index of patients in the corticosteroid sensitivity group and late corticosteroid resistance group. However, it promotes increased concentration of this substance in urine in the early corticosteroid resistance group. This led us to hypothesize that the mutation at rs $3738423(\mathrm{C}>\mathrm{T})$ is protective for patients responding to corticosteroid, however it will make early corticosteroid resistance patients soon fall into bad condition. If this polymorphism interacts with other genetic factors, further research is needed to test this hypothesis.

\section{Conclusions}

Urine biochemical index (protein, proteinuria/creatininuria index) and blood biochemical index (protein, albumin) are important indicators in the diagnosis and monitoring of childhood nephrotic syndrome. These parameters depend on the steroid response as well as on the interaction between this response and the genotype of NPHS2 rs3738423 (C>T) in patients with nephrotic syndrome.

\section{Acknowledgements}

We would like to thank the sponsorship of Vietnam National University, Hanoi for the topic of code number QG.16.23. Sincerely thank TS. Nguyen Thi Thu Huong, Department of Kidney Urology, Vietnam National Children's Hospital has supported the collection of blood samples and patient information. We would like to thank the Department of Basic Science in Medicine and Pharmacy, School of Medicine and Pharmacy, Vietnam National University, Hanoi; Department of Pediatrics, Department of Biochemistry, Hanoi Medical University collaborated on the topic.

\section{References}

[1] N Boute, O Gribouval, S Roselli, F Benessy, H Lee, A Fuchshuber, K Dahan, MC Gubler, P Niaudet and C Antignac. NPHS2, encoding the glomerular protein podocin, is mutated in autosomal recessive steroid resistant nephritic syndrome. Nature Genet. 2000; 24, 349-54.

[2] G Caridi, F Perfumo and GM Ghiggeri. NPHS2 (podocin) mutations in nephrotic syndrome, Clinical Spectrum and Fine Mechanism. Pediatr. Res. 2005; 57, 54R-61R. 
http://wjst.wu.ac.th

[3] HY Cho, JH Lee, HJ Choi, BH Lee, IIS Ha, Y Choi and HII Cheong. WT1 and NPHS2 mutations in Korean children with steroid resistant nephrotic syndrome. Pediatr Nephrol. 2008; 23, 63-70.

[4] H Junli. 2012. Genetics of Nephrotic Syndrome in Singapore Pediatrics Patients. Master Thesis. National University Singapore, Singapore.

[5] S Gulati, V Kher, RK Sharma and A Gupla. Steroid response pattern in Indian children with nephrotic syndrome. Acta Paediatr. 1994; 83, 530-3.

[6] J Mao, Y Zhang, L Du, Y Dai, W Gu, AM Liu, S Shang and L Liang. NPHS1 and NPHS2 gene mutations in Chinese children with sporadic nephrotic syndrome. Pediatr. Res. 2007; 61, 117-22.

[7] DTT Nga. 2011. Consideration on the Results of Treatment of Steroid Resistance in the Department of Kidney and Urology from the Vietnam National Children's Hospital. Master Thesis in Medicine. Hanoi Medical University, Hanoi, Vietnam.

[8] PTH Nhung, TVQ Giao, VV Nga, PV Dem, NTT Mau, DD Long and VT Thom. Initial results in genotyping of 6 exons of NPHS2 gene in pediatric patients with nephrotic synrome. VNU J. Sci. Med. Pharmaceut. Sci. 2017; 33, 60-4.

[9] H Otukesh, S Otukesh, M Mojtahedzadeh, R Hoseini, SM Fereshtehnejad, AR Fard, N Sadigh, AH Behzadi. R Javadi, N Hooman and M Mehrazma. Management and outcome of steroid-resistant nephrotic syndrome in children. Iran J. Kidney Dis. 2009; 3, 210-7.

[10] RR Roy, MR Islam, T Jesmin, A Matin and MR Islam. Prognostic value of biochemical and hematological parameters in children with Nephrotic syndrome. J. Shaheed Suhrawardy Med. Coll. $2013 ; \mathbf{5}, 95-8$.

[11] DT Tham. 2012. Consideration on the Results of Treatment of Treatment of Primary Nephrotic Syndrome in Children. Master Thesis in Medicine. Hanoi Medical University, Hanoi, Vietnam.

[12] LN Tra. Nephrotic Syndrome: Pediatric Lecture. Vol II. Medicine, Hanoi, 2009.

[13] A Vasudevan, A Siji, A Raghavendra, TS Sridhar and KD Phadke. NPHS2 Mutations in Indian Children with Sporadic Early Steroid Resistant Nephrotic Syndrome. Indian Pediatri. 2012; 49, 231-3.

[14] Z Yu, J Ding, J Huang, Y Yao, H Xiao, J Zhang, J Lui and J Yang. Mutations in NPHS2 in sporadic steroid resistant nephrotic syndrome in Chinese children. Nephrol. Dial Transplant. 2005; 20, 9028. 